\title{
An indirect route to repetitive actions
}

\author{
David M. Lovinger
}

Laboratory for Integrative Neuroscience, National Institute on Alcohol Abuse and Alcoholism, NIH, Bethesda, Maryland, USA.

It is increasingly evident that there is a genetic contribution to autism spectrum disorders (ASDs) and other neural disorders involving excessive repetition of action sequences. Among the implicated genes in these disorders are those encoding postsynaptic scaffolding proteins with roles in synaptic transmission and plasticity. Several mouse models harboring synonymous mutations have shown alterations in synaptic transmission within the striatum, which has key roles in controlling actions and action sequences. In this issue of the $J C l$, Wang and coworkers show that glutamatergic synaptic transmission onto striatal projection neurons is weakened in mutant mice lacking the $\mathrm{SH} 3$ and multiple ankyrin repeat domains 3 (SHANK3B) scaffolding protein, defective expression of which has been implicated in ASDs. This synaptic alteration gives rise to stronger activity in the indirect pathway accompanied by decreased dendritic spines on the indirect pathway medium spiny projection neuron, indicative of decreased numbers of glutamatergic synapses. Selectively enhancing activity in this pathway reduced excessive repetitive grooming in the mutant mice. Changes in glutamatergic input to striatal projection neurons have been observed in several other murine ASD models and associated disorders. Thus, manipulation of the function of the striatal indirect pathway may be a useful therapeutic target for treating disorders characterized by excessive repetitive behaviors.

\section{Excessive repetitive actions} in autism spectrum and other disorders

Excessive repetition of actions occurs in a variety of neural disorders, including autism spectrum disorders (ASDs) and other syndromes characterized by compulsion, such as addiction (1). Thus, there is widespread interest in determining the genetic and environmental factors that contribute to excessive actions as well as the underlying circuitry. Neuroscientists have developed mouse models expressing genetic alterations in proteins that have been linked to human disorders such as ASDs (2, 3). Among these are genes that encode postsynaptic scaffolding and cell-adhesion proteins with signaling functions (4-8). Synaptic transmission and the relationship to excessive actions are subjects of intense investigation in these genetic mouse models.

In this issue, Wang and coworkers report on their examination of the mechanisms that contribute to altered transmission and plasticity at glutamatergic synapses in the striatum of mice lacking the $\mathrm{SH} 3$ and multiple ankyrin repeat domains $3 \mathrm{~b}$ (SHANK3B) protein (9). Disrupted SHANK3 expression in humans has been implicated in ASDs $(10,11)$, and previous studies have shown that loss of this protein leads to excessive grooming in mice, one example of a repetitive/compulsive action (12-14). Moreover, Shank3-deficient mice show deficits in social interaction that mimic one component of human ASDs. Thus, understanding synapse and circuitry alterations in these

Related Article: p. 1978

mice could provide information relevant to neural dysfunctions in the human brain that underlie disorders of compulsion.

The striatum is a subcortical brain region implicated in the learning and control of actions (15). Alterations in neurons within the striatum and those that project to this brain region have been proposed to be involved in movement disorders such as Huntington's disease and Parkinson's disease (16). Afferent inputs from the cortex and thalamus to the striatum make glutamatergic synapses onto striatal neurons, providing excitatory input that drives the activity of these neurons $(17,18)$. The major targets of this excitatory drive are medium spiny neurons (MSNs) that provide output signals to other regions within the basal ganglia circuitry. The MSN efferent projections target two basal ganglia subregions. Projections to the substantia nigra pars reticulata directly inhibit this major basal ganglia output center; hence, this projection is termed the direct pathway (19). The net effect of direct pathway activation is increased activity of cortical neurons that promote action performance. The other major MSN efferent target is the globus pallidus external segment, which inhibits basal ganglia output, with a net effect of decreased cortical activity (19). This so-called indirect pathway contributes to selection of action sequences, perhaps by inhibiting unwanted sequences following cortical activity. Imbalances in direct and indirect pathway activity have been proposed as underlying several movement disorders and are also thought to be involved in compulsive behaviors (20-22). Alterations in synaptic input to the MSNs that give rise to these pathways could produce such imbalances, and there is increasing evidence of changes at striatal synapses in mouse models of neural disorders, including ASDs (5-8).

\section{Indirect pathway alteration} in SHANK3-deficient mice

Wang and coworkers used mice expressing genetically encoded fluorescent proteins to identify direct and indirect pathway MSNs in brain slices taken from mice 
A

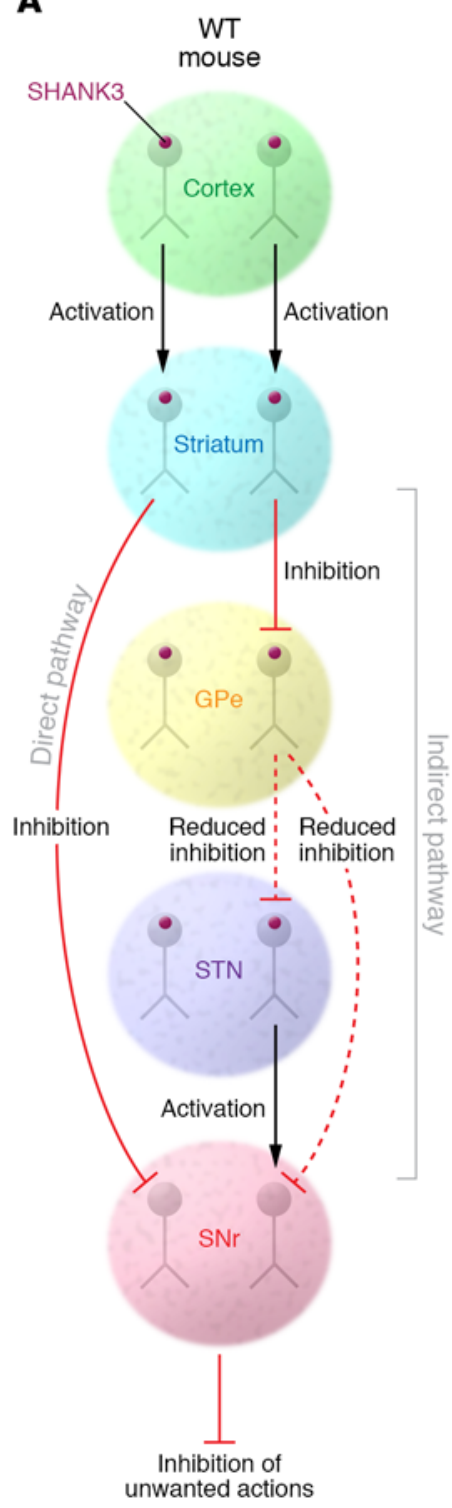

lacking the SHANK3 protein (9). Compared with those in control animals, glutamatergic synapses in Shank3-deficient mice showed decreased transmission in the indirect pathway MSNs, with no change in the synapses on direct pathway neurons. The authors also examined long-lasting changes in transmission at these glutamatergic synapses. Long-term depression (LTD) of synaptic transmission depends on activation of metabotropic glutamate receptors 1 and 5 (mGluR1 and mGluR5), L-type voltage gated calcium channels, and endocannabinoid activation of presynaptic cannabinoid 1 receptor (CB1R) (23). Wang and coworkers showed that LTD could not be induced in indirect pathway MSNs by
B

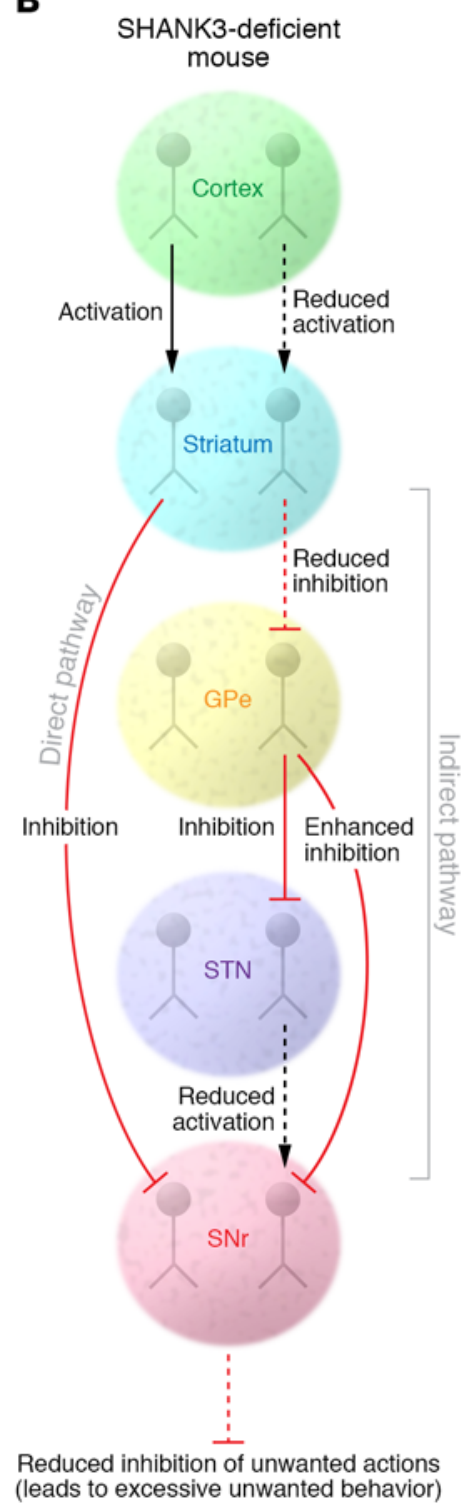

afferent stimulation or pharmacological activation of mGluRs or calcium channels in the SHANK3-deficient mice. In contrast, LTD was intact after all procedures in WT mice and in direct pathway MSNs in Shank3 mutant mice. These results support previous work indicating that LTD can be induced at glutamatergic synapses onto MSNs that give rise to both pathways (24). These changes in synaptic function were accompanied by a decrease in the number of spines on the dendrites of indirect pathway MSNs, where glutamatergic synapses are made by cortical afferents. This finding suggests that the number of glutamatergic synapses onto indirect pathway MSNs is decreased in the mutant mice and that reduction of
Figure 1. Decreased indirect pathway function in mice lacking the SHANK3 scaffolding protein. Schematic diagrams of the cortico-basal ganglia direct and indirect pathways in WT mice and mice lacking the SHANK3 postsynaptic scaffolding protein. In WT mice, cortical synaptic input to direct and indirect pathway MSNs helps to keep the appropriate balance of striatal output that determines which actions are allowed and which are suppressed. In mice lacking SHANKB, cortical input to indirect pathway MSNs is weakened, resulting in less synaptic excitation of these neurons and less CABAergic inhibitory output to the globus pallidus external segment (GPe). This results in greater GABAergic inhibition of GPe output to the substantia nigra pars reticulata $(\mathrm{SNr})$, thus suppressing basal ganglia output. The net result of these circuit changes is impaired suppression of unwanted actions, excessive grooming in particular. glutamatergic synapses could be a prominent mechanism underlying the decrease in synaptic transmission onto these projection neurons.

The reduction in glutamatergic synapses and synaptic transmission onto indirect pathway MSNs decreases excitation of these neurons in response to cortical (and perhaps thalamic) activity (Figure 1). The MSNs do not depolarize sufficiently to fire action potentials in the absence of coordinated glutamatergic synaptic transmission. Thus, decreasing the strength of this transmission would result in fewer indirect pathway MSNs being activated by bouts of glutamatergic input that occur during the initiation and performance of actions. When this effect is targeted primarily to 
indirect pathway MSNs, the net effect is a decrease in the striatal output to targets that normally reduce unwanted movements (25). Overall, the consequence of this circuit change would most likely be excessive production of certain actions. Such a mechanism could certainly contribute to the excessive bouts of grooming observed in SHANK3-deficient mice.

Indeed, Wang and coworkers demonstrated that activation of indirect pathway MSNs reduces excessive repetitive grooming in mice lacking Shank3. This was accomplished using a designer receptors exclusively activated by designer drugs (DREADD) approach, in which a clozapine- $N$-oxideactivated (CNO-activated) DREADD was specifically expressed in indirect pathway MSNs, resulting in increased MSN activity in mice given the drug. Reduced grooming was only seen in animals expressing DREADD in indirect pathway MSNs combined with drug treatment and not in CNO-treated animals that expressed DREADD in direct pathway MSNs. This finding supports the idea that abnormally strong indirect pathway activity contributes to excessive grooming in the mutant mice.

The loss of LTD observed in SHANK3deficient mice provides another mechanism through which glutamatergic transmission may be altered, leading to deficient indirect pathway activation. This type of synaptic plasticity has been implicated in the learning of actions (23). Thus, a sustained deficit in plasticity at these synapses may signal loss of normal action learning, further contributing to excessive, repetitive behaviors.

\section{Concluding remarks and future directions}

Additionally, the loss of SHANK3 throughout development in these mice could result in synaptic impairments that occur early in development that lead to later circuit and behavioral abnormalities. It is also possible that the relevant changes are part of ongoing synaptic adjustments that help to sustain the abnormal behavior. In this case, correcting the synaptic alterations in adult mice could restore normal behavior. A recent paper from the Feng laboratory showed that behavior, including excessive grooming, could be normalized by restoring SHANK3 expression in adult mice that lacked the pro- tein throughout development (6). Other behaviors, such as anxiety-like behaviors, were not rescued. The SHANK3-mediated behavioral rescue was accompanied by restoration of normal striatal synaptic transmission, although it was not determined whether this effect was specific to the indirect pathway. These findings indicate that lack of SHANK3 does not lead to irreversible developmental changes related to striatal function and excessive actions, providing hope that therapeutic approaches will benefit adults. However, sorting out the specific role played by SHANK3 deficiency-induced weakening of indirect pathway activity in adult animals with excessive repetitive behavior, as well as in humans, will be an important future research direction.

Alterations in glutamatergic striatal synaptic transmission have been observed in mouse models of several neural disorders that involve excessive repetitive and compulsive-like behaviors $(2,3)$, and the Feng laboratory has contributed many of these findings. One common emerging theme is that mutation of proteins involved in postsynaptic scaffolding, including SHANK3 and SAPAP3 (5), contributes to excessive repetitive behaviors associated with altered glutamatergic transmission in striatum. It will be important to determine whether indirect pathway MSNs are a common target in these mutant mouse models. Overall, a focus on impaired cortical excitatory drive to striatum appears to be an important area in research on ASD and other disorders of compulsion and may well be a focus for future therapeutic efforts.

\section{Acknowledgments}

This work was supported by the Division of Intramural Clinical and Biological Research of the NIAAA, project number ZIA AA000416 to DML. Karina Abrahao provided valuable assistance in the creation and refinement of the figure.

Address correspondence to: David M. Lovinger, Laboratory for Integrative Neuroscience, National Institute on Alcohol Abuse and Alcoholism, National Institutes of Health, 5625 Fishers Lane, Room TS-11, Bethesda, Maryland 20892, USA. Phone: 301.443.2445; E-mail: lovindav@ mail.nih.gov.
1. Voon V, Dalley JW. Translatable and back-translatable measurement of impulsivity and compulsivity: convergent and divergent processes. Curr Top Behav Neurosci. 2016;28:53-91.

2. Shepherd GM. Corticostriatal connectivity and its role in disease. Nat Rev Neurosci. 2013;14(4):278-291.

3. Monteiro P, Feng G. Learning from animal models of obsessive-compulsive disorder. Biol Psychiatry. 2016;79(1):7-16.

4. Espinosa F, Xuan Z, Liu S, Powell CM. Neuroligin 1 modulates striatal glutamatergic neurotransmission in a pathway and NMDAR subunit-specific manner. Front Synaptic Neurosci. 2015;7:11.

5. Welch JM, et al. Cortico-striatal synaptic defects and OCD-like behaviours in Sapap3-mutant mice. Nature. 2007;448(7156):894-900.

6. Mei Y, et al. Adult restoration of Shank3 expression rescues selective autistic-like phenotypes. Nature. 2016;530(7591):481-484.

7. Shmelkov SV, et al. Slitrk5 deficiency impairs corticostriatal circuitry and leads to obsessive-compulsive-like behaviors in mice. $\mathrm{Nat}$ Med. 2010;16(5):598-602.

8. Südhof TC. Neuroligins and neurexins link synaptic function to cognitive disease. Nature. 2008;455(7215):903-911.

9. Wang W, et al. Striatopallidal dysfunction underlies repetitive behavior in Shank3-deficient model of autism. J Clin Invest. 2017;127(5):1978-1990.

10. Wilson HL, et al. Molecular characterisation of the $22 \mathrm{q} 13$ deletion syndrome supports the role of haploinsufficiency of SHANK3/PROSAP2 in the major neurological symptoms. JMed Genet. 2003;40(8):575-584.

11. Durand CM, et al. Mutations in the gene encoding the synaptic scaffolding protein SHANK3 are associated with autism spectrum disorders. Nat Genet. 2007;39(1):25-27.

12. Bozdagi O, et al. Haploinsufficiency of the autism-associated Shank3 gene leads to deficits in synaptic 4 function, social interaction, and social communication. Mol Autism. 2010;1(1):15.

13. Peça J, et al. Shank 31 mutant mice display autistic-like behaviours and striatal dysfunction. Nature. 2011;472(7344):437-442

14. Wang $X$, et al. Synaptic dysfunction and abnormal behaviors in mice lacking major isoforms of Shank3. Hum Mol Genet. 2011; 20(15):3093-3108.

15. Steiner H, Tseng KY, eds. Handbook OfBasal Ganglia Structure And Function. London, United Kingdom: Academic Press/Elsevier; 2017.

16. Walters JR. Abnormal activities in cortic-basal ganglia circuits in movement disorders. In: Steiner H, Tseng KY, eds. Handbook OfBasal Ganglia Structure And Function. London, United Kingdom: Academic Press/Elsevier; 2017:741-754.

17. Reiner A, Deng Y. Organization of corticostriatal projection neuron types. In: Steiner H, Tseng KY, eds. Handbook Of Basal Ganglia Structure And Function. London, United Kingdom: Academic Press/Elsevier; 2017:405-422.

18. Galvan A, Villalba RM, Wichmann T, Smith Y. 
The thalamostriatal systems in normal and disease states. In: Steiner H, Tseng KY, eds. Handbook Of Basal Ganglia Structure And Function. London, United Kingdom: Academic Press/ Elsevier; 2017:477-494.

19. Gerfen CR, et al. D1 and D2 dopamine receptor-regulated gene expression of striatonigral and striatopallidal neurons. Science. 1990;250(4986):1429-1432.

20. Albin RL, Young AB, Penney JB. The functional anatomy of basal ganglia disorders. Trends Neurosci. 1989;12(10):366-375.

21. Chesselet MF, Delfs JM. Basal ganglia and movement disorders: an update. Trends Neurosci. 1996;19(10):417-422.

22. Tanimura Y, Vaziri S, Lewis MH. Indirect basal ganglia pathway mediation of repetitive behavior: attenuation by adenosine receptor agonists. Behav Brain Res. 2010;210(1):116-122.

23. Lovinger DM. Neurotransmitter roles in synaptic modulation, plasticity and learning in the dorsal striatum. Neuropharmacology. 2010;58(7):951-961.

24. Wang Z, et al. Dopaminergic control of corticostriatal long-term synaptic depression in medium spiny neurons is mediated by cholinergic interneurons. Neuron. 2006;50(3):443-452.

25. Mink JW. The basal ganglia: focused selection and inhibition of competing motor programs. Prog Neurobiol. 1996;50(4):381-425. 